\title{
CORRECTION OF CONCENTRATED AND DISTRIBUTED ABERRATIONS IN MEDICAL ULTRASOUND IMAGING
}

\author{
A.P.Berkhoff, J.M.Thijssen \\ Clinical Physics Laboratory, University Children's Center, \\ POBox 9101, NL-6500HB, Nijmegen, The Netherlands
}

\begin{abstract}
A method is presented for iterative correction of wave fields aberrated in a plane located at an arbitrary distance from an array transducer. The signals received from the transducer are processed by an inverse extrapolator in such a way that the output yields the transducer signals as if the transducer had been located directly at the position of the aberrator. For subsequent transmission cycles, the same inverse extrapolator is applied to delta pulses at time instants incorporating the time-reversed estimated aberration profile. The method can be applied to scattering and absorptive media, i.e. in medical conditions. The compensation of distributed aberration is also developed. It is shown that correction algorithms intended for concentrated aberrations can be used to reduce effects due to distributed aberrations; our conclusions with respect to the position of the equivalent concentrated aberrator differ from results reported in the literature. The method is demonstrated on realistic simulations of solid lesions, and cysts (voids) disturbed by intervening aberrating media.
\end{abstract}

\section{INTRODUCTION}

In medical ultrasonic imaging the interest is often found in tissue structures several hundreds of wavelengths away from the transducer. Distortions in intervening tissue lead to reduced image quality for the deeper lying structures in the region of interest. The distortions in intervening tissue can be so severe that there is virtually no information left of the region of interest. This makes subsequent tissue characterization procedures and video processing techniques based on signals from conventional beam formers a questionable activity.

Studies in ultrasonic beam degradation have led to a variety of correction and analysis methods. An early approach is the estimation of the arrival time in the receive aperture by cross-correlation techniques [1], [2]. Another approach is based on maximization of speckle brightness by adjusting the focusing delays [3]. A compromise between the full array optimization technique [3] and the adjacent element technique [1] was described by $\mathrm{Ng}$ et al. [4] as a partial array correction method or speckle look-back method. In the same paper [4] also a derivation was given for the equivalence of the adjacent element technique and the full array optimization technique. Gambetti and Foster [5] applied

This research was supported by grants from the Technology Branch of the Netherlands Foundation for Scientific Research and from Philips Medical Systems B.V., Best, The Netherlands. A.P.Berkhoff is currently with TNO-FEL, POBox 96864, NL-2509JG the Hague, the Netherlands. J.M.Thijssen is the corresponding author. E-mail.J.Thijssen@ckskg.azn.nl previous correction methods to annular array transducers. The aberrator profile can also be found by a least-squares fit of anival times [6] and computationally efficient correction methods were also given [7].

In all of the previous investigations, the implicit assumption was used that the aberrations occur directly in front of the transducer aperture. Considerable progress was obtained by Liu et al. [8], who showed that backpropagation methods in the temporal and spatial Fourier domain can be used to compensate time delay errors occuring at an arbitrary distance from the transducer.

In this paper, the latter backpropagation techniques are improved in several ways. Firstly, it is shown that, also in pulse-echo imaging situations, a proper backpropagation method in combination with an iterative scheme converges to the extraction of the desired aberration profile. Secondly, we use backpropagation before geometric correction in receive mode and backpropagation after geometric correction in transmit mode, instead of the reversed sequences to ensure that the methods obey the governing acoustic equations, such as the Helmholtz equation. Third, the backpropagation method described in this paper can also be used for media with absorption. Fourth, it is shown that the optimum location of an effective phase screen which represents an extended, distributed, random medium in a twoway point scatterer imaging configuration, is located more near to the transducer than to the region of interest, with the center of the aberrator as reference. This is in contrast with previously reported results [8]. Finally, the devised method might be used in digital echo scanners in which signals of the individual transducer elements are available for further processing.

\section{FORMULATION OF THE PROBLEM}

The basic geometry of the problem is shown in Fig. 1: an array transducer is geometrically focused at the region of interest (ROI) which contains a number of randomly positioned point scatterers. For each line contributing to the image, a subset of the total available transducer elements is used. An irregular acoustic interface between two media is located at a distance $d$ from the transducer. Due to the presence of the refractive interface, conventional beam forming 

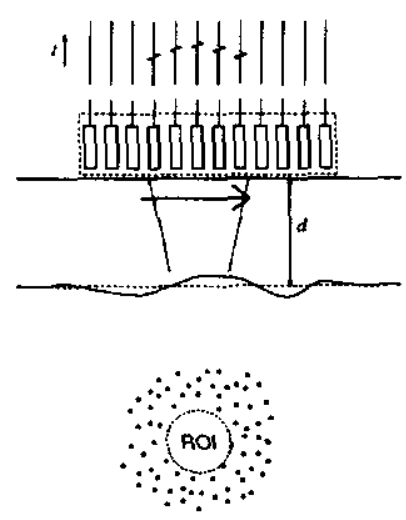

Fig. 1. Geometry showing an array transducer which employs a subset of the total number of elements for each scan line and where reflections from randomly positioned point scatterers contribute to the image. The wave fields are distorted by a refractive interface between two different acoustic media at an average distance $d$ from the transducer.

for transmitting and receiving can lead to distorted beams [9], [10], [11], and unsatisfactory images.

Considerable effort has been spent to reduce the image artifacts due to phase screens. A disadvantage of most existing methods, however, is that the phase screen is assumed to be directly in front of the transducer. Although some improvement still is possible if we assume a phase screen at $d=0$ where actually $d>0$, the solution is not optimal. Rather, we should try to compensate for the phase screen effects by making an educated guess for the distance $d$. This distance $d$ can be obtained in various ways, for instance from a clear reflecting surface in the image, a priori knowledge of subcutaneous tissue geometry, iterative search methods leading to a reduced image-based error criterion, etc.

Recently, a number of successive phase screens was used for describing wave propagation through human tissue [12], [13]. This leads to a distributed medium model where the distance between each of the phase screens should be on the order of the lateral correlation length [14]. However, there are several anatomical structures where a clear boundary between homogeneous (on a macroscopic level) tissue structures can be identified. For the latter cases a distributed model should be unnecessary. Furthermore, in this paper it will be shown that the image distortion due to a medium having distributed phase aberrations can be reduced effectively by assuming a single, more or less centered, phase screen. Optimum positions of the concentrated phase screen will be given. In addition, a relatively small number of these concentrated phase screens can be used to reduce distributed phase aberrations extending over a large thickness range.

\section{METHOD}

Three methods for the simulation of wave propagation through rough media interfaces were discussed in [15]. Two of these methods, the Conjugate Gradient methods, are rigorous, while the third, the phase screen method, is an approximation.

The approach we have followed is to apply the phase screen method in the initial development of the correction schemes. As a check of the phase screen simulations, the Conjugate Gradient Rayleigh method was used for providing more accurate simulations for a small number of image lines.

\section{Correlation method for aberration correction}

This subsection describes an implementation of an aberration correction method based on the correlation method of Flax and O'Donnell [1], [2]. In typical ultrasound systems, there is a high correlation between received signals at adjacent elements, even in the case of a medium consisting of a multitude of reflecting elements. This result was described in the van Cittert-Zernike theorem applied to ultrasound signals [16]. Because of the large similarity of adjacent element signals if phase distortion occurs directly at the transducer aperture, the location of the peak of the timedomain cross-correlation between these signals should lead to an accurate estimate of the relative time delay between the signals. In general, the cross-correlation $r_{x y}(t)$ of signals $x(t)$ and $y(t)$, where $t$ denotes the time coordinate, is efficiently calculated via the inverse Fourier transform of the frequency domain cross-correlation $R_{X Y}(\omega)$

$$
R_{X Y}(\omega)=X(\omega) Y^{*}(\omega)
$$

where $X(\omega)$ and $Y(\omega)$ are the Fourier transforms of $x(t)$ and $y(t)$, respectively, and the asterisk denotes the complex conjugate. One way to obtain the time delay from the crosscorrelation is to calculate the least-mean-square approximation of $\arg \left\{R_{X Y}(\omega)\right\}$ by a straight line through the origin [17]. We used the inverse Fourier transform of Eq. (1). In our application, we are interested in the relative time-delay between the signals of two adjacent transducer elements. Then we use the location $t=\tau_{i-1, i}$ of the maximum amplitude of the correlation function as the relative time delay between signals of transducer elements $i-1$ and $i$. Note that the location of the maximum of the correlation function corresponds to the relative time shift between the two signals which yields the minimum of the mean squared difference. The absolute time-delay $T_{i}$ of element signal $x_{i}(t)$, $i=1, \ldots I$ is given by the sum $T_{i}=\sum_{j=1}^{i} \tau_{j-1, j}$, where, by definition, $\tau_{0.1}=0$.

The aberration correction is applied after the conventional beamforming with geometrical focus at distance $F$. To ensure that the aberration correction process does not try to steer a beam to a different direction and depth than intended, the planar and constant component in the estimated time delays $T_{i}$ are removed by a least-squares detrending procedure. The resulting time delays $T_{i}^{\prime}$ can be used to align 
the signals from the receive beam former to create a single scan line. The same delays $T_{i}^{\prime}$ can also be used to create time-reversed pulses in order to anticipate the aberrating profile when transmitting in the following iteration. It was shown [1] that the iterative process of determining time delays from cross-correlation and re-emission of time-reversed signals converges and leads to the true aberrator profile.

\section{Correction for a distant phase screen}

In the previous subsection a method was discussed for the determination of an aberrator profile immediately in front of the transducer. In this subsection a method is given for the correction of a phase screen at a certain distance from the transducer. In order to get the desired results we calculate what the received signals would have been if the transducer were placed at the location of the aberrator. This allows us to align the signals with conventional phase aberration correction techniques which require the phase screen to be directly in front of the transducer for proper correction. In order to be able to perform the inversion we should know some medium properties and the distance $d$ to the aberrator. However, the guess for the aberrator distance $d$ is not critical, as are the medium properties which are also fairly constant in human tissue. Therefore, any reasonable guess for these parameters should lead to an improved result. The direct and weak forms of the angular spectrum decompensation applied in this study have been described by the author [15]. It appeared that evanescent waves can be neglected in this procedure and the method can be summarized as:

- The signals received from the transducer are transformed to the wavenumber-frequency frequency domain by the use of temporal and spatial Fourier transforms,

- The transformed representation is inversely extrapolated

- A geometrical focus corresponding to a distance of $F-d$ is applied,

- A cross-correlation and detrending procedure extracts the aberrator profile,

- A single scan line results from a summation in the time domain where the signals have been aligned according to the estimated aberrator profile,

- New transmit pulses are generated by applying the same inverse extrapolator to delta pulses at time instants according to the time-reversed estimated aberrator profile and shifts in time according to the geometrical focus at a distance $F-d$.

The complete sequence of operations is repeated until the estimated aberrator profile does not change anymore. A major computational task is the evaluation of temporal Fourier transforms and $n$-dimensional spatial Fourier transforms ( $n$ is the array dimensionality), which can be combined in more efficient $(n+1)$-dimensional Fourier transforms. It is shown in subsequent sections that the above procedure leads to the extraction of the true aberrator profile.
As was mentioned earlier, our procedure removes the planar component in the estimated aberration profile, which is equivalent to the removal of a steering term. This steering term should removed: otherwise every scan line would immediately "lock" on the strongest reflector. It is only necessary that the mean steering direction is kept the same as the intended direction. Therefore, removal of terms of order higher than one possibly leads to incomplete aberration correction.

\section{DISTRIBUTED ABERRATIONS}

When dealing with a medium which contains a distributed aberrator, it would be convenient to be able to use a lumped, concentrated, phase screen in the correction process. Assuming that it is possible to use such a concentrated phase screen, the question remains what the optimum location of the assumed phase screen should be. An error criterion is introduced to determine the optimum position.

\section{Desired properties of the concentrated phase screen}

If we know the extent and location of the distributed aberrator and assume the statistics of aberrator properties to be constant over the aberrator, then we can identify two desired properties of the assumed concentrated aberrator location. Firstly, if the concentrated aberrator location $d$ is further away from a slice $d z$ at depth $z$ of the distributed aberrator, then the approximation of the effects of that slice will be worse. Secondly, aberrators which are close to the transducer at depth $z=0$ have a much larger impact than aberrators which are near the region of interest at depth $z=F$. As a consequence, the correction algorithm should give priority to aberrations occuring in the near field. Note that this is consistent with previous results [14], where it was shown that a phase screen produces a halo of constant angular width $\theta$ rad around the main lobe. Then, the clutter integrated in the image is proportional to $(F-z) \theta$. These two considerations lead to an optimum concentrated phase screen position approximately at the center of the distributed aberrator.

\section{Waveform similarity and focusing criterion}

To obtain the optimum concentrated phase screen position, a criterion is needed that expresses the quality of the aberration correction. A suitable criterion for the focusing quality is in terms of the waveform similarity $r$ [8]

$$
r=\frac{\left(\int_{-\infty}^{\infty}\left(\sum_{i=1}^{I} x_{i}\left(t+T_{i}^{\prime}\right)\right)^{2} d t\right)^{1 / 2}}{\sum_{i=1}^{I}\left(\int_{-\infty}^{\infty} x_{i}^{2}(t) d t\right)^{1 / 2}}
$$

where the definition of the symbols can be found in section III. Mallart, who independently arrived at a similar expression [18], called it the focusing criterion $C$, where $C=r^{2}$. 


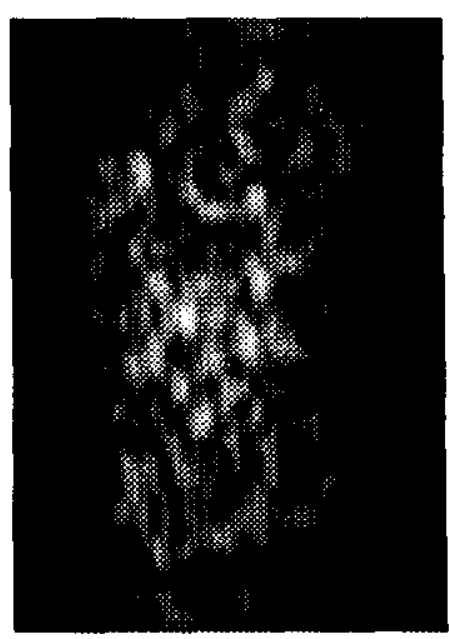

(a) No correction

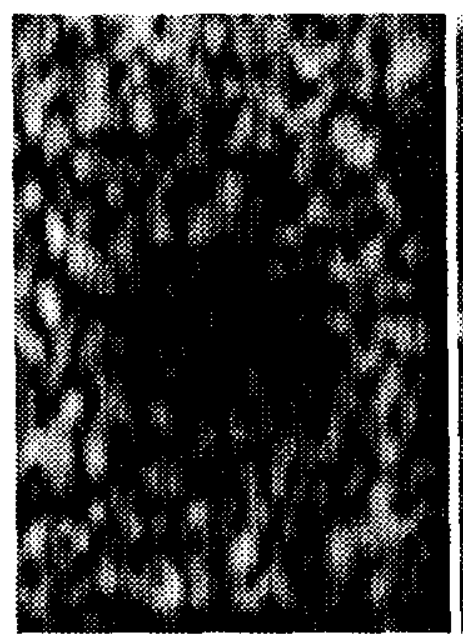

(d) No correction

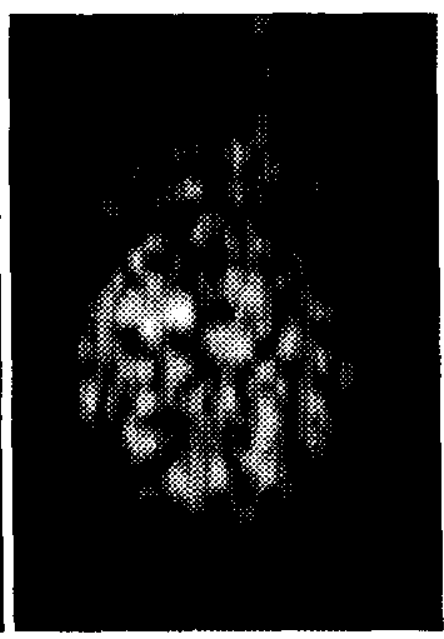

(b) Receive correction

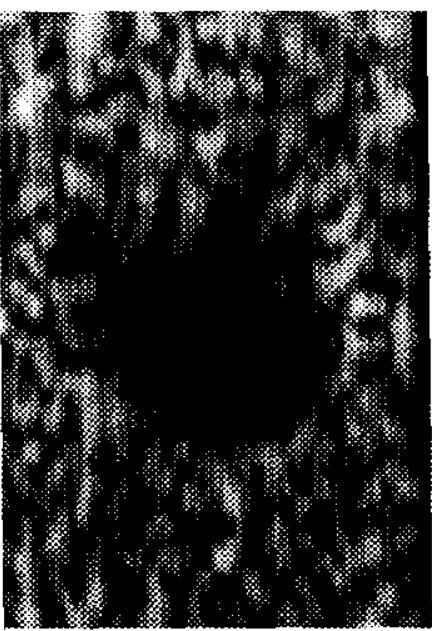

(e) Receive correction

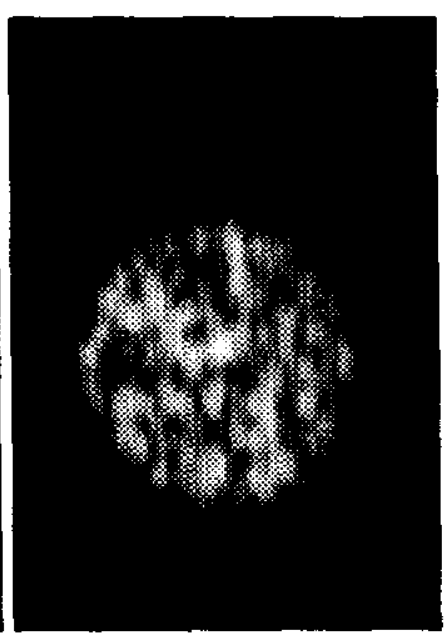

(c) Receive and transmit correction

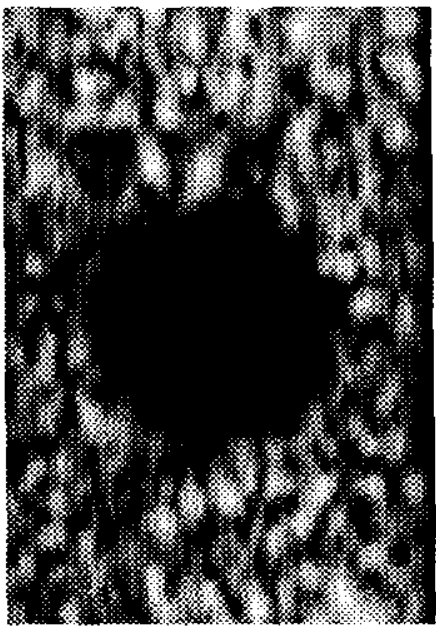

(f) Receive and transmit correction

Fig. 2. Image of a circular region of randomly positioned point scatterers (top row) and image of a void in a background medium containing randomly positioned point scatterers (bottom row) with aberrator at $z=10 \mathrm{~mm}$; backpropagation over $10 \mathrm{~mm}$.

It was shown that $C$ has a maximum value of 1 in case of a single point scatterer. In the case of a random distribution of point scatterers, however, the maximum value of $C$ is $2 / 3$. This value was confirmed by our simulations.

\section{RESULTS}

In this section the proposed correction method will be applied to simulated signals. The forward simulation problem of the homogeneous medium model with causal absorption has been described previously [19]. First the results will be given for the correction of a single phase screen. Thereafter, results for imaging through a succession of phase screens are given.

The background medium has a zero frequency sound speed $c_{0}=1540 \mathrm{~m} / \mathrm{s}$ and density $\rho=10^{3} \mathrm{~kg} / \mathrm{m}^{3}$. The absorption in the medium is $0.5 \mathrm{~dB} /(\mathrm{cm} \mathrm{MHz})$. The transducer is assumed to have a Gaussian function describing the transfer from voltage to surface velocity. The central frequency is 3.5 $\mathrm{MHz}$ with a $-6 \mathrm{~dB}$ bandwidth of $2.25 \mathrm{MHz}$. The total number of transducer elements is 128 at an element pitch of $0.4 \mathrm{~mm}$ and an element width of $0.3 \mathrm{~mm}$. The aperture for each scan line consists of a group of 64 transducer elements, 


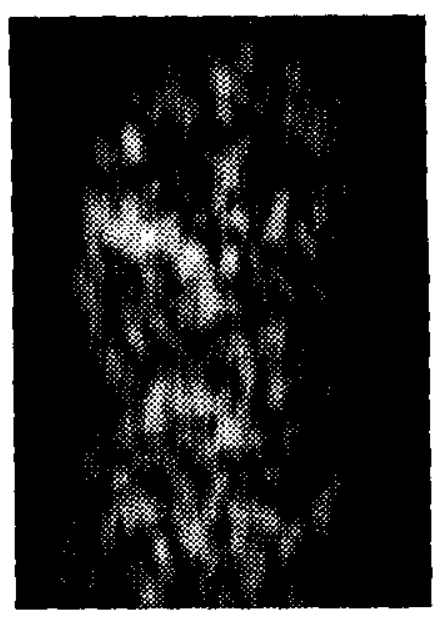

(a) no aberration correction

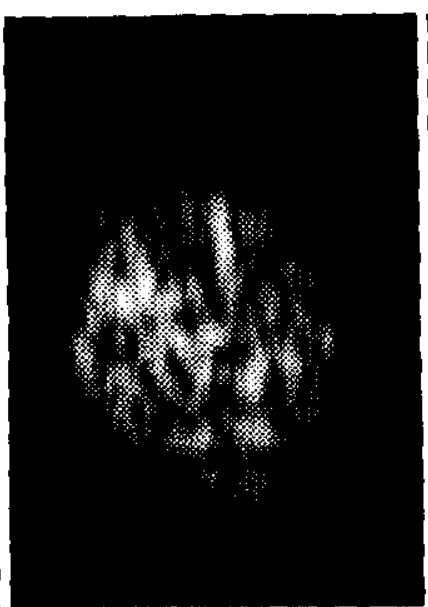

(b) Effective phase screen at 0 mm

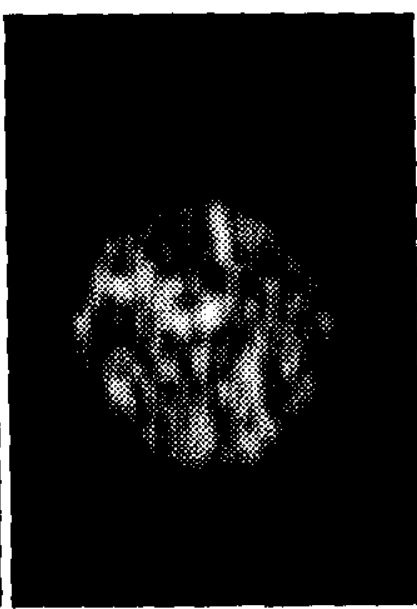

(c) Effective phase screen at 10 $\mathrm{mm}$

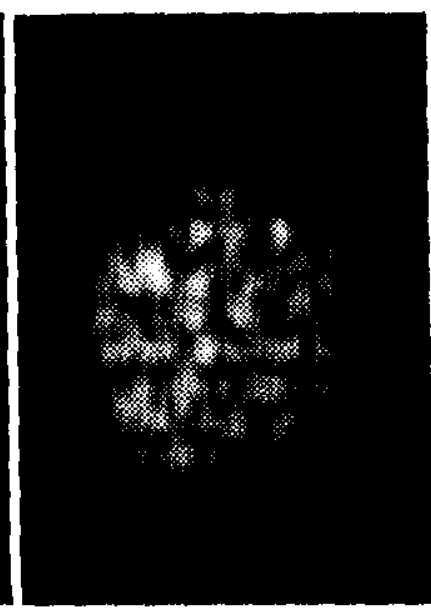

(d) Effective phase screen at 15 mm

Fig. 3. Image of circular region containing randomly positioned point scatterers for a distributed aberrator between $z_{1}=5 \mathrm{~mm}$ and $z_{2}=15 \mathrm{~mm}$. Aberration correction has been applied in receive and transmit mode; an effective concentrated phase screen was assumed for the correction process.

which results in a maximum of 65 scan lines. In the images only 36 scan lines are shown, however. All the targets to be imaged are located or centered at a distance of $50 \mathrm{~mm}$ from the array. As targets are used: a circular region with point scatterers, and a circular void without point scatterers. The sampling frequency is $50 \mathrm{MHz}$, which is used throughout the scheme. In the images the transducer is positioned to the left. The images have a size of $14.4 \mathrm{~mm}$ (lateral) $\times 10$ $\mathrm{mm}$ (depth) and are displayed with a gamma compression factor 2 .

\section{Imaging through a concentrated aberrator}

In this subsection results are shown for the aberration correction method in case the aberrator consists of a single, concentrated, phase screen. The distance of the phase screen to the transducer is $10 \mathrm{~mm}$. The phase screen has an rms time-shift of 100 ns. The lateral characteristics of the phase screen are described by a Gaussian correlation function with a correlation distance of $2 \mathrm{~mm}$.

The first target is a circular region with a diameter of 6 $\mathrm{mm}$ containing randomly positioned point scatterers in an otherwise reflection-free medium. In Fig. 2a the result without aberration correction is shown. Fig. $2 \mathrm{~b}$ shows the result if aberrations are corrected for only the received signals. In Fig. $2 c$ we have re-emitted transmit pulses based on a time-reversed aberrator profile obtained from the previous receive aberration estimation.

The iterative aberration correction also works for a void target, which is defined as a circular region with a diameter of $6 \mathrm{~mm}$ in which scatterers are absent. The background medium contains randomly positioned point scatterers. In
Fig. $2 d$ the result without aberration correction is shown. In Fig. 2e the image is shown if we apply an aberration correction only to the received signals. Fig. 2f shows the image if we re-emit transmit pulses based on a time-reversed aberrator profile obtained from the previous receive aberration estimation. It can clearly be seen that also in this case the images improve in the iteration process.

\section{Imaging through a distributed aberrator}

The correction method described previously is able to exactly compensate for the concentrated aberrator if the distance $d$ is known. In practice we either do not know exactly the position of a (possibly) concentrated aberrator or we are dealing with a truly distributed aberrator. To show the performance of the correction algorithm for more or less realistic situations as in the latter two cases, we will use a distributed aberrator. The distributed aberrator consists of a sequence of five phase screens with a thickness of $2 \mathrm{~mm}$, extending over depth $z_{1}<z<z_{2}$ in which $z_{1}=5 \mathrm{~mm}$ and $z_{2}=15 \mathrm{~mm}$. Each individual phase screen has a lateral correlation length of $2 \mathrm{~mm}$ with Gaussian characteristics and an rms time shift of $40 \mathrm{~ns}$. In Fig. 3a the resulting image is shown if no correction is applied.

Fig. 3b-d show results for three backpropagation distances. Only the results after one re-emission are given. If the concentrated aberrator position is taken to be the center of the distributed aberrator, we get $d=10 \mathrm{~mm}$. Fig. $3 \mathrm{~b}-\mathrm{d}$ show the results for: no backpropagation, $10 \mathrm{~mm}$ backpropagation, and $15 \mathrm{~mm}$ backpropagation, from left to right, respectively. Results are shown for the normal backpropagator instead of the weak backpropagator. The target to clutter 
ratio TCR [20] is $19 \mathrm{~dB}, 25 \mathrm{~dB}$ and $23 \mathrm{~dB}$, respectively. Thus the backpropagation to the center of the distributed aberrator gives the best result. The result for $15 \mathrm{~mm}$ backpropagation is also quite good. The conclusion is that the assumed effective phase screen distance is not critical, because all three distances, $0 \mathrm{~mm}, 10 \mathrm{~mm}$ and $15 \mathrm{~mm}$, lead to considerably improved images as compared to Fig. 3a. For a backpropagation distance of $15 \mathrm{~mm}$, bad results were obtained with the weak form of the backpropagation operator, with a TCR as low as $10 \mathrm{~dB}$

The results of Liu and Waag [8] indicate that the optimum concentrated aberrator position is more near to his point-like source than to the receiving array. This conflicts with the results reported here. It is believed that the difference has to be attributed to the use of the fourth-order polynomial in the aberration correction procedure by Liu. The subtraction of a fourth-order term leads to insufficient removal of important lower-order aberration components.

\section{REFERENCES}

[1] S.W. Flax and M. O'Donnell, "Phase-aberration correction using signals from point reflectors and diffuse scatterers: basic principles," IEEE Trans. Ultrason. Ferroelect. Freq. Control 35, 758-767, 1988.

[2] M. O'Donnell and S.W. Flax, "Phase-aberration correction using signals from point reflectors and diffuse scatterers: measurements," IEEE Trans. Ultrason. Ferroelect. Freq. Control 35, 768-774, 1988.

[3] L. Nock, G.E. Trahey and S.W. Smith, "Phase aberration correction in medical ultrasound using speckle brightness as a quality factor," J. Acoust. Soc. Am. 85, 1819-1833, 1989.

[4] G.C. Ng, S.S. Worrell, P.D. Freiburger and G.E. Trahey, A comparative evaluation of several algorithms for phase aberration correction," IEEE Trans. Ultrason. Ferroelect. Freq. Contr. 41, 631-643, 1994.

[5] C. Gambetti and F.S. Foster, "Correction of phase aberrations for sectored annular array ultrasound transducers," Ultrasound Med. Biol. 19, 763-776, 1993.

[6] D.-L. Liu and R.C. Waag, "Time-shift compensation of ultrasonic pulse focus degradation using least-mean square error estimates of arrival time," $\mathrm{J}$. Acoust. Soc. Am. 95, 542-555, 1994.

[7] M. Karaman, A. Atalar, H. Kömen and M. O'Donnell, "A phase aberration correction method for ultrasound imaging," IEEE Trans. Ultrason. Ferroelect. Freq. Contr. 40, 275-282, 1993.

[8] D.-L. Liu and R.C. Waag, "Correction of ultrasonic wavefront distortion using backpropagation and a reference waveform method for time-shift compensation," J. Acoust. Soc. Am. 96, 649-660, 1994.

[9] A.P. Berkhoff, P.M. van den Berg and J.M. Thijssen, "Iterative calculation of reflected and trans- mitted acoustic waves at a rough interface," IEEE Trans. Ultrason. Ferroelect. Freq. Control 42, 663$671,1995$.

[10] L.A.F. Ledoux, A.P. Berkhoff and J.M. Thijssen, "Ultrasonic wave propagation through aberrating layers: experimental verification of the Conjugate Gradient Rayleigh method," IEEE Trans. Ultrason. Ferroelect. Freq. Control 43, 158-166, 1996.

[11] A.P. Berkhoff, P.M. van den Berg and J.M. Thijssen, "Ultrasound wave propagation through rough interfaces: iterative methods," J. Acoust. Soc. Am, 99, 1306-1314, 1995.

[12] Q. Zhu and B.D. Steinberg, "Wavefront amplitude distribution in the female breast," J. Acoust. Soc. Am. 96, 1-9, 1994.

[13] Q. Zhu and B.D. Steinberg, "Modeling, measurement and correction of wavefront distortion produced by breast specimens," proc. Ultrasonics Symposium, IEEE New York, Ed. M. Levy, 1613-1617, 1994.

[14] B.D. Steinberg, "Scattering from a multiple random random phase screen model of a random inhomogeneous medium," J. Acoust. Soc. Am., submitted, 1996.

[15] A.P. Berkhoff, "Ultrasonic imaging in aberrating media: simulation methods for the design of aberration correction schemes," $\mathrm{PhD}$ Thesis, Nijmegen University, The Netherlands, 1996.

[16] R. Mallart and M. Fink, "The van Cittert-Zernike theorem in pulse echo measurements," J. Acoust. Soc. Am. 90, 2718-2717, 1991.

[17] J.S. Bendat and A.G. Piersol, Engineering Analysis of Correlation and Spectral Analysis, New York: Wiley-Interscience, 1980.

[18] R. Mallart and M. Fink, "Adaptive focusing in scattering media through sound-speed inhomogeneities : The van Cittert-Zernike approach and focusing criterion. J. Acoust. Soc. Am. 96, 3721-732, 1994.

[19] A.P. Berkhoff, J.M. Thijssen and R.J.F. Homan, "Simulation of ultrasonic imaging with linear arrays in causal absorptive media," Ultrasound Med. Biol. 22, 245-259, 1996.

[20] D.H. Turnbull, P.K. Lum, A.T. Kerr and F.S. Foster, "Simulation of B-scan images from twodimensional transducer arrays: Part I - Methods and quantitative contrast measurements," Ultrasonic Imaging 14, 323-343, 1992. 